Article

\title{
Detecting the Visible: The Discursive Construction of Health Threats in a Syndromic Surveillance System Design
}

\author{
Baki Cakici $^{1,2, *}$ and Pedro Sanches ${ }^{1,3}$ \\ ${ }^{1}$ Swedish Institute of Computer Science (SICS), Kista 164 29, Sweden; E-Mail: sanches@ sics.se \\ ${ }^{2}$ Department of Computer and Systems Sciences, Stockholm University, DSV, Kista 164 40, Sweden \\ ${ }^{3}$ Royal Institute of Technology (KTH/ICT/SCS), Kista 164 40, Sweden \\ * Author to whom correspondence should be addressed; E-Mail: cakici@ sics.se; \\ Tel.:+46-8-633-1500.
}

Received: 31 March 2014; in revised form: 11 July 2014 / Accepted: 21 July 2014 /

Published: 28 July 2014

\begin{abstract}
Information and communication technologies are not value-neutral tools that reflect reality; they privilege some forms of action, and they limit others. We analyze reports describing the design, development, testing and evaluation of a European Commission co-funded syndromic surveillance project called SIDARTHa (System for Information on Detection and Analysis of Risks and Threats to Health). We show that the reports construct the concept of a health threat as a sudden, unexpected event with the potential to cause severe harm and one that requires a public health response aided by surveillance. Based on our analysis, we state that when creating surveillance technologies, design choices have consequences for what can be seen and for what remains invisible. Finally, we argue that syndromic surveillance discourse privileges expertise in developing, maintaining and using software within public health practice, and it prioritizes standardized and transportable knowledge over local and context-dependent knowledge. We conclude that syndromic surveillance contributes to a shift in broader public health practice, with consequences for fairness if design choices and prioritizations remain invisible and unchallenged.
\end{abstract}

Keywords: ICT; public health surveillance; epidemiology; syndromic surveillance; discourse 


\section{Introduction}

The term syndromic surveillance describes a collection of methods within the field of public health surveillance. These are characterized by their use of secondary sources, referring to data collected for a variety of purposes only indirectly related to population health. For example, over-the-counter medicine sales, or records of ambulance dispatches from a hospital, or records of emergency room visits can be considered secondary sources, because these data are not collected with the primary intent to perform public health surveillance. Using this formulation, syndromic surveillance is often positioned as an efficient use of already collected data [1].

International public health institutions rely increasingly on automated and globalized surveillance, and this shift follows a rationale of vigilance and preparedness where "marginal and dangerous" [2] events are given priority over broader determinants of health [3]. Within the discourse of syndromic surveillance, numerical representations of populations and the application of statistical analyses are essential to the task of constructing meaning from diverse sources of surveillance data. The use of these types of surveillance methods follows a long tradition of health discourses where individuals and populations are constructed as knowable and governable by establishing comparative systems to analyze their similarities and differences [4] (p. 190).

Equally important is the idea of disease as a collection of symptoms and signs observed by a medical gaze [5] (pp. 88-106). The idea of extending the medical gaze and the multiplication of ways of representing individuals and populations are not exclusive to public health institutions. Two other analyses included in this special issue also deal with systems of classification and comparison, which make use of similar discourses. In the two studies, these systems have consequences for how motherhood is performed [6] and how people reflect about themselves [7].

We analyze reports describing the design, development, testing and evaluation of a European Commission co-funded syndromic surveillance project called SIDARTHa (System for Information on Detection and Analysis of Risks and Threats to Health). We start with the assumption that information and communication technologies (ICTs) are never value-neutral. They necessarily reflect the viewpoints of their designers, the influence of others involved in their construction and the consequences of the material limitations encountered during their development [8-10]. With our analysis, we aim to identify how a syndromic surveillance discourse privileges or excludes specific types of knowledge and practices. We show that the discourse of syndromic surveillance constructs the health threat concept as a sudden, unexpected event with the potential to cause severe harm and one that requires a public health response aided by surveillance. Based on our analysis, we state that when creating surveillance technologies, design choices have consequences for what can be seen and for what remains invisible. Finally, we argue that syndromic surveillance discourse privileges expertise in developing, maintaining and using software within public health practice, and it prioritizes standardized and transportable knowledge over local and context-dependent knowledge. We conclude that syndromic surveillance contributes to a shift in broader public health practice, with consequences for fairness if design choices and prioritizations remain invisible and unchallenged. 


\section{Background}

The earlier applications of syndromic surveillance were characterized by the use of health-related data that preceded diagnosis and the continual monitoring of disease indicators to detect outbreaks of communicable diseases earlier than traditional methods [11-13]. More recently, the definition of syndromic surveillance has broadened to include the monitoring of non-communicable diseases and other health conditions, such as heat-related illnesses, injuries caused by tornadoes or respiratory illness after wildfires [14].

A well-known example of syndromic surveillance is Google Flu Trends [15,16], which uses search queries entered by users into Google as indicators of influenza outbreaks. The developers of the system have claimed that they can "accurately estimate the current level of weekly influenza activity in each region of the United States" [15] (p. 1012), although the estimates have not always been accurate [17,18]. While the system itself is well-known, it is highly unusual, as it is run by a private company. The majority of syndromic surveillance systems are used by public institutions, such as regional and national health authorities.

Many different types of ICTs are used for communicable disease surveillance. Bravata et al. [19] provide a broad review of 115 systems and identify those that provide syndromic signals. Other reviews focus on syndromic surveillance and include more recent systems [20,21]. Additionally, the Triple-S project provides an extensive list of syndromic surveillance systems used within Europe [22].

\subsection{Challenges}

The impact of syndromic surveillance on the already limited resources available for public health, for example the costs of dealing with potentially large numbers of false positives generated by these systems, has been discussed by public health practitioners and scholars. One of the earliest challenges was by Reingold [23] who asked: "If syndromic surveillance is the answer, what is the question?" Reingold points to the scarce resources within the field of public health and challenges those who call for the further development of syndromic surveillance to detail not how detection could be improved, but if public health response would be improved in any way if these systems are implemented. He emphasizes the importance of building capacity within the public health departments instead of collecting and analyzing data externally, an activity often performed by for-profit corporations in departments without academic partners. In a discussion on the difficulties of creating a nation-wide syndromic surveillance, Mostashari voices concerns about the relationship between detection and response capacity:

We have 80 percent of the nation covered but we really have nothing covered, because signals come and go, and an e-mail maybe is sent out, and there's no local capacity ... If you're doing analysis for a thousand different towns, villages, cities, whatever, every day you're going to find alarms. ([24]; cited in [25])

Similarly, Heffernan et al. [26] state that although their syndromic surveillance systems have been beneficial in detection, they are "essentially 'smoke detectors' and call for prompt investigation and response if they are to provide early warning of outbreaks" [26]. They continue to recommend that 
efforts for bioterrorism preparation should focus on hiring well-trained public health professionals with responsibilities that are broader than just bioterrorism.

Fearnley [25] analyzes the debates surrounding the development and use of several nation-wide syndromic surveillance systems in the U.S. and identifies a central conflict: the early syndromic surveillance systems were designed primarily for national bioterrorism preparedness, but the data collection and reporting were assigned to public health institutions tasked with maximizing the health of human populations. These systems collected data from many different, non-traditional sources and transferred them to health departments. However, the collected data were not immediately helpful, because " $[\mathrm{m}]$ ore information means more interpretive work, without certain benefits; and more detected events requires more epidemiological responses, without (at this point) the necessary epidemiological resources to undertake them" [27] (p. 84). Fearnley points out that without the resources to interpret the incoming data, the newly developed systems were not helpful to epidemiologists.

In syndromic surveillance systems, collecting data is the key to tackling public health problems. All syndromic surveillance systems are constructed with the assumption that gathering more data helps public health authorities make decisions. Analyzing the practices that accompany ICT-based surveillance systems in public health, French [28] identifies the assumption that more data are helpful as a consequence of an immaterial conception of information:

[...A]n immaterial conception of information, whether implicit or explicit, assumes that information signifies in the same way regardless of time or place. As a consequence of this assumption the significance of information-processing practices is minimized; the material diversity of such practices, and the effort required to unify them, is underestimated. French [28] (p. 111).

As French argues, data collection also comes at a cost. It requires significant amounts of work to standardize data, to connect systems and to ensure that data are transferred correctly from one place to another. Ignoring the cost of collection or, more broadly, the material dimensions of information, causes problems for ICTs. The very immediate consequence is that it requires more work. This is the work of maintaining and interpreting.

In their extensive study of classification, Bowker and Star point out that data entry is never a trivial task: it requires trained staff to perform; it is prone to mistakes with respect to the classifications being used, and there are always cultural variations in what is interpreted as worthy of recording and what is omitted [29] (p. 107). Additionally, they state that there is always a tension between the standardization of lists centrally, and their use locally [29] (p. 139). Finally, they emphasize that "all category systems are moral and political entities" [29] (p. 324). As syndromic surveillance systems rely heavily on rigid classification schemes, they exhibit these characteristics.

Based on a study of medical records, Berg and Bowker also argue that data collection and recording methods influence how work is organized and which practices are considered to be part of that work [30] (p. 532). Similarly, emphasizing data collection and information generation in public health surveillance modifies the definition of activities that belong to public health, and some types of expertise are prioritized over others. For example, when syndromic surveillance is used, familiarity with developing and maintaining software becomes an important requirement for public health practice. 
The concept of immutable mobiles, introduced by Latour [31] (p. 227), provides a way to engage the processes of informatization and standardization in syndromic surveillance. Latour uses the term to refer to objects that are archivable and comparable regardless of their age, place of origin and context of use. For example, an entry in a table listing the admissions to the emergency room can be considered an immutable mobile, because, unlike the locally bound emergency room, it can be transported easily, compared to other descriptions, or even be organized in charts and statistics to construct an aggregated view of events that occur in emergency rooms. The complexity of the emergency room, or any other phenomenon, is overcome by translating it into intelligible and stable objects. The power of these objects is tied to their simplicity, which makes complex phenomena observable and manageable from a bird's-eye view. For this reason, immutable mobiles allow power-the power to see and the power to manage - to be exerted, irrespective of time and place.

However, the work of keeping these objects stable is often unaccounted for. In fact, the structures that maintain them, such as the layout of the rows and columns in a table or the procedure for filling out the details of each entry, like the objects, must also remain constant across places and contexts for them to remain immutable. The concept of immutable mobile is descriptive, allowing us to identify and describe certain objects in our analysis. We focus on how they are created and how they are maintained. We then take a step further by problematizing the aspects of their creation and maintenance. The immutability of these objects can only be accomplished by mobilizing people and material resources around them and by keeping these networks together through constant work [31]. For example, the output of the peripheral surveillance systems are brought closer to each other by categorizing syndromes, collecting data and creating graphs, but constant work is required to uphold the categorizations. Given the costs and risks of immaterial conceptions of information [28] and the work of standardization, we ask the question of who benefits or what there is to be gained.

\section{Analysis}

SIDARTHa is designed to be installed at health institutions to monitor sources of emergency data, including records of emergency dispatches and reports from emergency practitioners. We chose to analyze the SIDARTHa project, as it is one of the largest syndromic surveillance systems developed in Europe, and it involves multiple partners from different member states. Similar projects that focus on single health institutions separately instead of one centralized system have also been developed in the U.S., such as RODS (Real-Time Outbreak and Disease Surveillance) [32]. Although the majority of syndromic surveillance systems in the last decade have been developed and used in the U.S., we consider ourselves more qualified to analyze a European project due to our previous experience in public health surveillance in an EU member state [33].

Our analysis is based primarily on the seven project reports published by the SIDARTHa project between the years 2009 and 2010. They describe the process of design, development, testing and evaluation of the SIDARTHa syndromic surveillance system. These reports form a suitable corpus for discourse analysis, because the authors describe different stages of the project in detail, and they state their reasons for their decisions. The earliest reports focus on literature reviews and pre-studies; later reports describe the design and development of the system, and the most recent ones discuss testing and 
evaluation. This progression allows us to observe changes and shifts in the project and see how different ideas evolve throughout the design and development of the system.

We began our analysis by reading the seven project reports and marking all sentences that make knowledge or truth claims related to the overall argument, that state a hypotheses or that present research questions. We continued by marking sentences that inform the reader about the existence of entities outside of the text. In this category, we included any statements that refer to uncertainty, because such statements also provide insight into what is considered to exist only partially outside the text. We also marked all sentences that refer to individuals or groups of people, because these statements show how the text constitutes subject positions that are relevant to its own argument. After this process was completed, we read only the marked sentences for each report and tried to group them according to common themes based on either the topics they discuss or the frequency of the words they use. We examined these themes in relation to knowledge [34] (p. 14), truth [35] (pp. 92-96), [36] (p. 12) and subject positions [36] (p. 15). We repeatedly asked the question "what is considered true in this statement?" and wrote down our interpretations, while keeping in mind the identified themes for each report, the collection of marked sentences, as well as the unmarked sentences referenced by the marked ones.

\subsection{What is a Health Threat?}

In the SIDARTHa reports, the aim of the project is stated as detecting public health threats [37] (p. 1). The reports further describe their goal as helping public health authorities to become aware of health threats earlier or even to prevent them. The scope of the term health threat within the project reports is very broad: it refers to diseases, both communicable and non-communicable [37] (p. 1), but it also refers to other events, such as floods, heat waves and even volcanic eruptions [38]. Additionally, it can refer to acts of bioterrorism, which most commonly refer to the intentional release of biological agents, such as anthrax, into the air in large quantities with the intent to harm others [37] (p. 7).

From the perspective of syndromic surveillance systems, health threats first become visible when other surveillance systems capture their traces. For example, a person experiencing high fever and a sore throat may visit the emergency room, where her visit is recorded in the admission logs, or several people living in the same district might post on social media platforms about suffering from shortness of breath and digestive problems. In both cases, the experiences of these individuals are recorded, and those records are then accessed by syndromic surveillance systems. For these systems, the health threat is best defined in terms of data and signals: "Early detection of public health threats in general relies on the components: timely and reliable data; the sensitivity, specificity; and timeliness of signals detected." [39] (p. 3).

Syndromic surveillance relies on the presence and the functioning of other surveillance systems. For example, the primary motivation for tracking ambulance dispatches is not to generate signals for syndromic surveillance. Ambulances are dispatched to retrieve patients, and the signals are used for a variety of purposes. Ambulance drivers may need to track their working hours, or hospital administrators may need to know how many ambulances are available to respond in case of emergencies, etc. Every departure and arrival of the ambulance is logged for a multitude of reasons, and syndromic surveillance 
systems depend on these logs, but the logs themselves are not produced specifically, or at least not primarily, to support the practice of syndromic surveillance.

The SIDARTHa reports describe a system that can be used to collect emergency data in different health institutions in multiple European countries independent of one another, each with their own rules and regulations for gathering data. To form a more homogeneous set of records, the designers propose a coding standard to convert the local data to the SIDARTHa-compatible version. Organizationally, the data remains locally stored, as one of the aims of the system is to ensure that the collected data does not leave the collecting institution or regional authority.

The standardized data format is a string of numbers with different fields. The designers state that "[t]he minimum data set for syndromic surveillance must contain enough information to produce the number of cases per day for temporal syndromic surveillance" [40] (p. 6). It includes seven variables: the anonymous case identifying number, date, geographic reference, syndrome, age, gender and severity. The final three variables are called modifiers, because these are not essential to constructing the number of cases, but the information contained within them may be relevant for certain syndromes, such as gastroenteritis (or stomach flu) in children or heat-related illness in the elderly. These particular variables are chosen based on a survey of availability within the countries that participate in the SIDARTHa project, and it is the minimal set of properties that all of the participants are able to provide.

Using the standard, it is possible to represent each field using numbers, even when receiving data from different sources. For example, the case identification can be filled using the call identification code if the data are provided by an emergency medical dispatch center, or it can be filled using the patient identification code if the provider is an emergency department. The date is converted to a series of unambiguous numbers by specifying the order that day, month and year appear in the string. Geographic reference is more heterogeneous; the numbers contained in this field can refer to $\mathrm{X}$ and $\mathrm{Y}$ coordinates generated using the Global Positioning System (GPS), health zone codes, post codes or community codes. Attempting to fit gender into this form brings the ubiquitous problem of coding gender as a binary and the transition from sex to gender in the system vocabulary. The original specification of the standard provides " 0 " for male and " 1 " for female, and the final implementation of the system changes the order and adds a third category, "unknown" [41] (p. 33).

All of the variables we have described above pertain to the identification of persons and their locations. The actual work of describing syndromes occurs in the remaining two variables: syndrome and severity. The syndrome category requires the largest amount of work to convert into a number. Syndromes are divided into six categories: influenza-like, gastrointestinal, respiratory, intoxication, environment-related (heat-related) and unspecific. Since the system is designed to be compatible with many different sites, a multitude of conversion tables are provided to make possible the homogeneous coding of cases for any SIDARTHa system. The coding manual provides a series of tables to allow this conversion, where the codes of other, more established standards, such as the International Classification of Diseases (ICD), can be converted to the SIDARTHa standard [40].

At the end of this conversion, the health threat is narrowed from a wide variety of uncontrollable events to a series of numbers that can be transmitted without change and one that refers to the same thing regardless of context. Unlike the data collected in each institution, which carry marks of local practices particular to each case, the resulting string is constructed to function independently of its context. It is 
easily comparable and combinable across different databases. In Latour's terms, this standardization creates an immutable mobile [31] that can be used for statistical analysis in any institution. Freed from their earlier contexts, these strings of numbers gain a comparability that the contextually-rich data available at the institutions do not possess. Once they are created by the SIDARTHa software, their comparability is put to use through the work of different classification and detection algorithms.

The classification may indicate change over time, or it may include a spatial component that connects the data points to particular places using GPS coordinates or postal codes. Although the algorithms vary depending on the input and the intent, the primary purpose of this analysis is to divide the data points into two groups: expected and unexpected. The data points that end up in the expected category are not of high importance to the system designers. These may indicate low-risk diseases in low volumes or a seasonal variation in an illness (implying that the change occurs every year). The designers of SIDARTHa are much more interested in the unexpected category. This category includes all of the data points that have been marked by the system as deviating from the norm. These unexpected events are all potential health threats within the system.

The designers state that one of SIDARTHa's strengths is its flexibility: "[T]he SIDARTHa system can easily be adjusted to cover additional health threats, in this case the volcanic ash cloud with new syndromes such as traffic accidents and cardiovascular syndrome." [42] (p. 10). This flexibility is not only due to the way the system has been constructed, but also because of how the discourse of syndromic surveillance describes health threats. In another report, they point out that "[o]ne important feature of syndromic surveillance is flexibility, which allows the generation and monitoring of syndromes according to suddenly emerging, potential health threats" [39] (p. 3). In this definition, a health threat is a sudden event that has the potential to affect population health. The words "suddenly" and "potential" highlight the issue of the absent subject in the definition: for whom is the event sudden or unexpected, and what is meant by the potential to affect? The concern about sudden events can be viewed from the perspective of public health authorities who would be expected to respond to an event. Suddenness can then be stated relative to how long it would take for the authorities to act (by contacting the patients, by visiting the clinics, by issuing public notices, etc.) when the health threat appears. Defining the limits of the word "potential" are much more difficult. For example, an influenza outbreak can sometimes spread rapidly in a population, but result in only minor suffering as the infected experience the discomfort of a sore throat. However, it can also cause major illness in those who are infected and even become life-threatening for those who already suffer from other conditions. Complicating the decision even further, the difference between the two is often not clear until its effects are experienced by individuals. The public health response is required to negotiate this conflict and to come to a decision about the limits of the potential of a disease to affect the population.

In the SIDARTHa reports, a particularly unusual event serves to illustrate the potential usefulness of the system for public health response, even in cases of non-detection. Following the eruption of the Eyjafjallajökull volcano in Iceland in 2010, the project consortium developed a method to evaluate the potential public health impact of the ensuing ash cloud. The event is described as offering a suitable scenario for testing the system, although the authors also point out:

This report uses the term 'volcanic ash cloud' without determining if the ash cloud was a cloud or rather a contamination. Therefore, it should be understood that the term 'ash cloud' 
used throughout this report is not to be understood as a scientific term. It should be further noted that the authors do not intend to give any prejudice on the question if there was any risk to health at all caused by the ash cloud as such. The intention of this rapid assessment was to test the capability of the SIDARTHa concept and pilot syndromic surveillance system to be timely adjusted for monitoring a suddenly occurring event potentially affecting health [emphasis in the original] [38] (p. 3).

A sudden event and its potential to affect health both appear in this justification for performing the ash cloud assessment using the SIDARTHa system. Establishing an event as a health threat occurs before the investigation begins, but the results of the investigation do not fully resolve the status of the event either. In the four SIDARTHa implementation sites in Austria, Denmark, Germany and Spain, the system identifies no significant correlation between the volcanic ash cloud and the unusual signals of respiratory conditions reported during the same period. The authors state that "further in-depth analysis of case characteristics is necessary" [38] (p. 16), but also mention that their investigation, and the fact that it was possible to perform it at all, demonstrates the "flexibility of syndromic surveillance systems to be used for ad-hoc surveillance after suddenly occurring events" [38] (p. 16). In the booklet Generic Public Health Preparedness in Europe, a brief description of the SIDARTHa project is accompanied by several quotes from Dr Thomas Krafft, the scientific-technical coordinator of the project. Commenting on the volcano investigation, he states:

The European Centre for Disease Prevention and Control asked us to test out SIDARTHa during this time to see if there were any health impacts from the volcanic ash plume .... We found no increased demand for emergency care services. It is important to be able to distinguish between 'real threats' and 'perceived threats' [43].

This division between real and perceived threats positions SIDARTHa, and by extension, syndromic surveillance, as the arbiter of truth for public health practice in the implementation sites. The operations of SIDARTHa are also perceptions, although they are perceptions of expertise, supported by advanced ICTs and public health authorities. The "real" in the quote can be interpreted as describing events that public health authorities should act upon to improve the health of a population; the "perceived" can also be interpreted as events that they should act upon to reassure the population that there is no health effect. In either case, the detection possibilities offered by SIDARTHa are geared towards shaping the type of public health response following an event.

In the beginning, the traces of different individuals are collected in various public health surveillance systems and converted into the SIDARTHa standard to create a more uniform unit that is compatible with the different methods of statistical analysis. Up until this point, the traces of individuals shed their context until only the bare minimum remained. The contents of that bare minimum are defined by the SIDARTHa standard. After reaching that point, the work of inscribing a new context into the numbers begins. The first step is the statistical analysis, which constructs the objects that public health institutions can act on, followed by the visualization of the results, which show the traces in the unexpected category. The process that begins with the set of recorded traces and eventually becomes the evidence for the existence or the non-existence of the health threat depends on a long chain of translations between different systems and different mediums. When the authors assert their claim as "[e]mergency 
care demand shows a pattern allowing for detection of unusual aberrations from the expected demand" [44] (p. 20), they refer to the large web of surveillance systems that individually construct and link the patterns, detections, aberrations and expectations.

\subsection{Design Choices and Expertise}

The SIDARTHa system uses emergency care data to monitor health threats. The project researchers divide it into four data sources: emergency medical service (EMS), emergency medical dispatch (EMD), emergency physician service (EP) and emergency department (ED) data. Each source has different properties that make it more or less suitable for inclusion in a syndromic surveillance system. For example, the authors note that ED data are often collected electronically, while EMS data are filled in paper forms that are then transferred to the computer, making the latter more difficult to connect to an ICT-based surveillance system [37] (p. 20). The authors also mention that emergency data covers only severe cases, because patients with mild symptoms are not very likely to call the emergency medical service or to visit an emergency department [37] (p. 22). At the same time, emergency departments receive patients and respond to calls outside the working hours of other health services [37] (p. 22).

The decision to use emergency data for syndromic surveillance has consequences for the types of events that can be detected and for those likely to remain invisible. In this case, one consequence is being able to detect severe cases and not being able detect mild ones. For example, the authors state that "since gastrointestinal problems do not mainly lead to the need of emergency medical care treatments, these outbreaks can only be identified by a syndromic surveillance system if the outbreak occurs under special circumstances (i.e., symptoms in a group from abroad)" [42] (p. 15). The system itself makes events that manifest with acute symptoms more visible. This is in line with the vision of syndromic surveillance as a tool of preparedness for sudden, unexpected and highly dangerous events. By beginning with the aim of detecting health threats and then setting up a system that is suited to the detection of severe illness rather than mild illness, the designers contribute to the definition of a health threat. In the previous examples, the health threat was a sudden event with the potential to affect health, whereas now a health threat is specified further as an event that can cause severe illness with sharp and sudden symptoms.

During the development of the SIDARTHa system, the ICT company, BeValley, programmed the system to match the specifications of the designers. In the evaluation report, the authors state that "BeValley agreed to adjust and update the system in the future, but the question remains how this can be sustained also with additional funding" [42] (p. 10). They also ask: "If the regional system cannot easily be repaired by the future users, how does that affect the usefulness and acceptance of the system?" [42] (p. 11). The authors anticipate that future users in emergency care institutions will have ICT staff who can install the software and program the data transfer [42] (p. 11), but they do not elaborate on either whether emergency care institutions do in fact have such capacity or if that capacity should be found within emergency care. The expertise required to perform public health surveillance grows when SIDARTHa or a similar syndromic surveillance system is introduced, and that new practice privileges ICT expertise more than before.

Finally, the work of classifying and sorting does not end with the programming and installation of the system. During the data analysis study, Rosenkötter et al. [39] report numerous errors that 
need to be resolved locally by the practitioners attempting to perform analyses based on emergency data. For example, the authors find that it is not possible to analyze unspecific syndromes using the Austrian emergency physician service data due to repeated entries for the same incident, while using the emergency department data, the only option is to analyze unspecific syndromes, because the source does not list the reasons for care. The Belgium emergency physician service data arrive with delay, causing difficulties in developing a syndrome-specific coding, and the emergency department data is not possible to analyze fully, because the systems recording the data have changed recently. The authors also describe the necessity of taking into account ICD shortlists used by physicians at different sites. These lists lump the detailed categories of the ICD into larger groups, allowing the physicians to quickly assign codes without referring to the extensive ICD documentation every time. However, the mappings of these shortlists differ from site to site, and they do not combine easily when centralizing the data. It is not possible to ignore the shortlists either, because similar cases may be assigned to different syndromes if the mappings in the shortlists are not taken into consideration. Although these events may sound like exceptions to the rule of smooth classification, as Bowker and Star [29] point out, the work of classification always includes these complexities and tensions.

\section{Conclusions}

We have demonstrated how a discourse of syndromic surveillance constructed the health threat concept as a sudden, unexpected event with the potential to cause severe harm and one that requires a public health response. Based on our analysis of reports from the SIDARTHa project, we stated that when creating surveillance technologies, design choices have consequences for what can be seen and for what remains invisible. We argued further that syndromic surveillance discourse privileges expertise in developing, maintaining and using software within public health practice, and it prioritizes standardized and transportable knowledge over local and context-dependent knowledge.

ICTs are not value-neutral tools; they privilege some forms of action, and they limit others. They are imbued with values, and different people benefit or suffer from their use differently. Latour's definition of technology as "society made durable" [45] underlines the potential of ICTs to stabilize existing social processes. Leaving design decisions unexamined can perpetuate current epidemiological classifications, which may already be problematic [46]. Furthermore, Fearnley states that "[s]yndromic surveillance itself, with its orientation towards unexpected events and nonspecific objects, inevitably moves epidemiology in new directions." [27] (p. 84). French's analysis of ICT use within public health defines some of these new directions:

[...A]n over-arching immaterial conception of information imbues some kinds of information with more import, for surveillance, than other kinds of information. Specifically, this conception encourages the collection of abstract, digitized signifiers, while simultaneously marginalizing other kinds of embodied, contextual information. Indeed, the pursuit of immaterial information for public health surveillance produces a dominant but superficial epidemiology at the expense of other potentially more effective epidemiologies. [28] (p. 6)

Syndromic surveillance systems are not costless solutions, because gathering information and sustaining surveillance require work. They also require a different kind of expertise not always found 
in public health institutions. Moreover, syndromic surveillance relies on a particular definition of health threat, which focuses on catastrophes and rare events. The practice of syndromic surveillance contributes to a shift in broader public health practice, and although this is not problematic in itself, since practices can always change, the question remains: what does syndromic surveillance mean for fairness? If it orients public health towards the detection of catastrophes or sudden events with severe consequences, what happens to other types of events that do not produce such signals? For example, to what extent can syndromic surveillance deal with chronic illness or aid those who suffer from health issues due to poverty? Public health practice is a large field, and there may be a place for different surveillance systems that monitor different types of illness. However, discussions of syndromic surveillance should not stop at sensitivity, specificity and timeliness, but also address questions of fairness.

\section{Author Contributions}

Baki Cakici collected the textual material. The authors performed the analysis and drafted the manuscript together. Both authors read and approved the final manuscript.

\section{Conflicts of Interest}

The authors declare no conflict of interest.

\section{References}

1. Mandl, K.D.; Overhage, J.M.; Wagner, M.M.; Lober, W.B.; Sebastiani, P.; Mostashari, F.; Pavlin, J.A.; Gesteland, P.H.; Treadwell, T.; Koski, E.; Hutwagner, L.; et al. Implementing Syndromic Surveillance: A Practical Guide Informed by the Early Experience. J. Am. Med. Inform. Assoc. 2004, 11, 141-150.

2. Weir, L.; Mykhalovskiy, E. Global Public Health Vigilance: Creating a World on Alert; Routledge: New York, NY, USA, 2010.

3. French, M. Woven of War-Time Fabrics: The Globalization of Public Health Surveillance. Surveill. Soc. 2009, 6, 101-115.

4. Foucault, M. Discipline \& Punish: The Birth of the Prison; Vintage: New York, NY, USA, 1995; originally published in 1977.

5. Foucault, M. The Birth of the Clinic: An Archaeology of Medical Perception; Vintage: New York, NY, USA, 1994; originally publihsed in 1973.

6. Johnson, S.A. "Maternal Devices", Social Media and the Self-Management of Pregnancy, Mothering and Child Health. Societies 2014, 4, 330-350.

7. Ruckenstein, M. Visualized and Interacted Life: Personal Analytics and Engagements with Data Doubles. Societies 2014, 4, 68-84.

8. Winner, L. The Whale and the Reactor: A Search for Limits in an Age of High Technology; University of Chicago Press: Chicago, IL, USA, 1986.

9. Albrechtslund, A. Ethics and Technology Design. Ethics Inf. Technol. 2007, 9, 63-72.

10. Manders-Huits, N. What Values in Design? The Challenge of Incorporating Moral Values into Design. Sci. Eng. Ethics 2011, 17, 271-287. 
11. Mostashari, F.; Hartman, J. Syndromic Surveillance: A Local Perspective. J. Urban Health 2003, 80, i1-i7.

12. Buehler, J.W.; Hopkins, R.S.; Overhage, J.M.; Sosin, D.M.; Tong, V. Framework for Evaluating Public Health Surveillance Systems for Early Detection of Outbreaks. MMWR Morbid. Mortal. Wkl. Rep. 2004, 53, 1-11.

13. Henning, K.J. What is Syndromic Surveillance? MMWR Morbid. Mortal. Wkl. Rep. 2004, $53,7-11$.

14. Buehler, J.W.; Whitney, E.A.; Smith, D.; Prietula, M.J.; Stanton, S.H.; Isakov, A.P. Situational Uses of Syndromic Surveillance. Biosecur. Bioterror: Biodef. Strat. Pract. Sci. 2009, 7, $165-177$.

15. Ginsberg, J.; Mohebbi, M.H.; Patel, R.S.; Brammer, L.; Smolinski, M.S.; Brilliant, L. Detecting Influenza Epidemics Using Search Engine Query Data. Nature 2009, 457, 1012-1014.

16. Google. Google Flu Trends. Available online: http://www.google.org/flutrends (accessed on 28 March 2014).

17. Cook, S.; Conrad, C.; Fowlkes, A.L.; Mohebbi, M.H. Assessing Google Flu Trends Performance in the United States during the 2009 Influenza Virus A(H1N1) Pandemic. PLoS One 2011, doi:10.1371/journal.pone.0023610.

18. Butler, D. When Google Got Flu Wrong. Nature 2013, 494, 155-156.

19. Bravata, D.M.; McDonald, K.M.; Smith, W.M.; Rydzak, C.; Szeto, H.; Buckeridge, D.L.; Haberland, C.; Owens, D.K. Systematic Review: Surveillance Systems for Early Detection of Bioterrorism-Related Diseases. Ann. Internal Med. 2004, 140, 910-922.

20. Chen, H.; Zeng, D.; Yan, P. Infectious Disease Informatics: Syndromic Surveillance for Public Health and Bio-Defense; Springer: Berlin, Germany, 2009.

21. Cakici, B. Disease Surveillance Systems. Royal Institute of Technology (KTH). Licentiate Thesis. Available online: http://kth.diva-portal.org/smash/record.jsf?pid=diva2:416962 (accessed on 24 July 2014).

22. Triple-S. Human Health Projects and Systems Identified Through the Triple-S Inventory. Available online: http://syndromicsurveillance.eu/systems-in-europe/humansys (accessed on 28 March 2014).

23. Reingold, A. If Syndromic Surveillance is the Answer, What is the Question? Biosecur. Bioterror: Biodef. Strat. Pract. Sci. 2003, 1, 77-81.

24. USMI. Weaving a National Surveillance System-The Role of Federal Healthcare, Technical Report; US Medicine Institute for Health Studies: Washington, DC, USA, 2003.

25. Fearnley, L. Signals Come and Go: Syndromic Surveillance and Styles of Biosecurity. Environ. Plann. A 2008, 40, 1615-1632.

26. Heffernan, R.; Mostashari, F.; Das, D.; Karpati, A.; Kulldorff, M.; Weiss, D. Syndromic Surveillance in Public Health Practice, New York City. Emerg. Infect. Dis. 2004, 10, 858-864.

27. Fearnley, L. Redesigning Syndromic Surveillance for Biosecurity. In Biosecurity Interventions: Global Health and Security in Question; Lakoff, A., Collier, S.J., Eds.; Columbia University Press: New York, NY, USA, 2008; pp. 61-88. 
28. French, M. Picturing Public Health Surveillance: Tracing the Material Dimensions of Information in Ontario's Public Health System. Ph.D. Thesis, Queen's University, Kingston, Ontario, Canada, 2009.

29. Bowker, G.C.; Star, S.L. Sorting Things Out: Classification and Its Consequences; The MIT Press: Cambridge, MA, USA, 2000.

30. Berg, M.; Bowker, G. The Multiple Bodies of the Medical Record: Toward a Sociology of an Artifact. Sociol. Q. 1997, 38, 513-537.

31. Latour, B. Science in Action: How to Follow Scientists and Engineers Through Society; Harvard University Press: Cambridge, MA, USA, 1988.

32. Espino, J.U.; Wagner, M.; Szczepaniak, C.; Tsui, F.C.; Su, H.; Olszewski, R.; Liu, Z.; Chapman, W.; Zeng, X.; Ma, L.; et al. Removing a Barrier to Computer-based Outbreak and Disease Surveillance: The RODS Open Source Project. MMWR Morbid. Mortal. Wkl. Rep. 2004, 53, 32-39.

33. Cakici, B.; Hebing, K.; Grünewald, M.; Saretok, P.; Hulth, A. CASE: A Framework for Computer Supported Outbreak Detection. BMC Med. Informat. Decis. Making 2010, 10, 14. doi:10.1186/1472-6947-10-14.

34. Mills, S. Discourse; Routledge: New York, NY, USA, 2004.

35. Foucault, M. The Will to Knowledge: The History of Sexuality Volume 1; Penguin: London, UK, 1998; originally published in 1978.

36. Jorgensen, M.W.; Phillips, L.J. Discourse Analysis as Theory and Method; SAGE Publications Ltd.: London, UK, 2002.

37. Early Detection of Health Threats: International State-of-the-Art \& European Health Surveillance Landscape. Results from the SIDARTHa Project; Ziemann, A., Krafft, T., Garcia-Castrillo Riesgo, L., Fischer, M., Krämer, A., Lippert, F., Vergeiner, G., Eds.; SIDARTHa Project: Bad Honnef, Germany, 2009.

38. SIDARTHa Volcanic Ash Cloud Rapid Public Health Impact Assessment. Regional Public Health Impact of Volcanic Ash Cloud Covering Europe after Eruption of Eyjafjallajoekull, Iceland Starting April 14th, 2010. Results as of May 15th, 2010; Rosenkötter, N., Ziemann, A., Garcia-Castrillo Riesgo, L., Vergeiner, G., Fischer, M., Krafft, T., Brand, H., Lippert, F., Krämer, A., Pinheiro, P., Eds.; SIDARTHa Project: Bad Honnef, Germany, 2010.

39. Retrospective Data Analysis and Simulation Study as Basis for an Automated Syndromic Surveillance System. Results from the SIDARTHa project; Rosenkötter, N., Kauhl, B., Garcia-Castrillo Riesgo, L., Llorca Diaz, F.J., Kraan, J., Ziemann, A., Schorbahn, M., Krafft, T., Brand, H., Eds.; SIDARTHa Project: Bad Honnef, Germany, 2010.

40. The SIDARTHa Coding Manual-How to Generate Syndromes Based on Routinely Collected Emergency Care Data for the European Syndromic Surveillance System SIDARTHa; Garcia-Castrillo Riesgo, L., Rosenkötter, N., Ziemann, A., Krafft, T., Fischer, M., Lippert, F., Vergeiner, G., Krämer, A., Brand, H., Eds.; SIDARTHa Project: Bad Honnef, Germany, 2009.

41. SIDARTHa Technical Guidelines. How to Implement the European Emergency Data-based Syndromic Surveillance System SIDARTHa; Garcia-Castrillo Riesgo, L., Ziemann, A., Krafft, T., 
Conde, R., Fischer, M., Vergeiner, G., Lippert, F., Krämer, A., Pinheiro, P., Brand, H., Eds.; SIDARTHa Project: Bad Honnef, Germany, 2010.

42. SIDARTHa Syndromic Surveillance System: Test, Evaluation \& Recommendations; Pinheiro, P.; Ziemann, A.; Rosenkötter, N.; Krämer, A.; Garcia-Castrillo Riesgo, L., Krafft, T., Fischer, M., Vergeiner, G., Lippert, F., Brand, H., Eds.; SIDARTHa Project: Bad Honnef, Germany, 2010.

43. EU Health Programme. Generic Public Health Preparedness in Europe; Brochure; European Union, 2011; doi:10.2772/59355. Available online: http://ec.europa.eu/eahc/documents/health/ leaflet/generic_preparedness.pdf (accessed on 24 July 2014).

44. Using Emergency Care Data for Syndromic Surveillance in Europe. Public Health Threats Detectable by and Availability of Routinely Collected Emergency Care Data in Europe. Results from the SIDARTHa Project; Baer, M., Ozguler, A., Garcia-Castrillo Riesgo, L., Krafft, T., Fischer, M., Krämer, A., Pinheiro, P., Lippert, F., Vergeiner, G., Brand, H., Eds.; SIDARTHa Project: Bad Honnef, Germany, 2009.

45. Latour, B. Technology is Society Made Durable. In A Sociology of Monsters: Essays on Power, Technology, and Domination; Law, J., Ed.; Routledge: New York, NY, USA, 1991; pp. 103-131. 46. Lupton, D. Epidemiology as a Sociocultural Practice. Crit. Public Health 1997, 7, $28-37$.

(c) 2014 by the authors; licensee MDPI, Basel, Switzerland. This article is an open access article distributed under the terms and conditions of the Creative Commons Attribution license (http://creativecommons.org/licenses/by/3.0/). 